\title{
The expanding new era of cancer immunotherapy
}

\author{
Elias A. Kotteas MD, PhD, Konstantinos N. Syrigos MD, PhD, FCCP \\ Oncology Unit, 3rd Department of Internal Medicine, \\ Athens School of Medicine, Sotiria General Hospital, Athens, Greece
}

Received 14 February 2016; Accepted 28 March 2016

Fighting cancer through the strengthening of the immune system has long been though an attractive and promising way to devising effective therapies. In the immunosuppressive milieu of cancer microenvironment created by ligands and coinhibitory receptors, tumorinfiltrating lymphocytes are ineffective and unable to exert their specific functions. Although it has been shown that immune cells could promote cancer progression via the growth of clones not susceptible to anticancer immunity, immunotherapy as an option for cancer treatment, despite initial setbacks, has altered the well-known paradox, i.e., foreign molecules from cancer cells to be viewed as self. Nevertheless, tumor characteristics give way to strategies to dysregulate the effectiveness of T-cell mediated attacks, such as the attenuation of antigenpresenting cells, the suppression of effector lymphocytes through the activation of immunosuppressive cells, and the establishment of a barrier at the vasculature that prevents homing of effector-rejecting cells.

Over the last decade, we have witnessed a paradigm shift in the treatment of cancer. Many ways have been identified in which the immune system may be manipulated to attack cancer cells including the reduction of immunosuppression and T-cell modulation and augmentation of adaptive immunity leading to cancer immunosurveillance. Recent studies have shown that cancer cells are often infiltrated by CD8 cells that produce better outcomes through the acquisition of control over cancer cells. The antitumor immune response is a multistep process performed by effector T-cells that recognize and kill tumor targets. Usually, at the tumor site, APC's take up antigens to be processed. Using dendritic cells, it is possible to present antigens in a way that stimulates $\mathrm{T}$ regulatory cells, which would oppose an antitumor response. APCs require a maturation signal in order to promote immunity. Partial maturation may be induced through toll-like receptor signaling from necrotic tumor cells.

The successful use of checkpoint inhibitors led to a breakthrough in the development of cancer immunotherapy. The anti-CTLA-4 monoclonal antibody Ipilimumab was the first checkpoint inhibitor that achieved durable objective responses in metastatic melanoma. The anti-PD-1 monoclonal antibodies Nivolumab and Pembrolizumab have demonstrated effective results in a variety of solid tumors such as nonsmall cell lung cancer, renal cancer, and melanoma. An additional advantage of using these agents emerges from the fact that these drugs often target the immune system and not the cancer. The combined administration of these antibodies has produced impressive results in melanoma patients. The enhancement of anticancer immunity and the control of the immune suppressive environment is an important issue for the development of cancer immunotherapy. Cancer immunology research has focused on studies regarding the establishment of the immune-suppressive environment and on the identification of molecules and cells involved in these pathways. In addition, the adoptive transfer of tumor-specific autologous T-cells using T-cells with cloned T-cell receptors or chimeric antigen receptor has led to substantial clinical responses. There appears to be a correlation between the number of somatic mutations detected by whole exome sequencing and response to immunotherapeutic agents. This may be a result of T-cell recognition of the tumor to T-cell exhaustion. Checkpoint inhibitors turn the T-cell recognition back on, leading to higher rates of prolonged survival benefit. As the repertoire of 
checkpoint inhibitors continues to move through various stages of clinical development, we will hopefully gain a deeper understanding of how checkpoint inhibitors work, how to best utilize the agents, and which patients will benefit most of them.

Chemotherapeutic agents were thought to be immunosuppressive, and recent studies have demonstrated that some of them may induce an immunogenic form of cancer cell death that augments anticancer immune responses. The success of these combined approaches may be partially explained by the crosstalk between cancer cells, tumor stroma, and the patient's immune system. Major considerations exist for the implementation of chemoimmunotherapyefficient combinations. For patients with metastatic cancer, a single therapeutic modality is highly unlikely to achieve long-term outcomes and immunotherapy should be combined with conventional chemotherapy. Patients who could benefit from such combinations must be carefully selected using biomarkers, which also emphasize the need for predictive biomarkers that can be introduced. Additionally, the choice of combinations, the dosage, and the schedule of administration remain to be determined. The main issue is how to integrate the immunotherapy treatments with the standard clinical strategy. Future trials will sequence cancer vaccines, immunomodulators, and conventional cancer treatments.

More recently, it has emerged that the tumor vasculature constitutes an important barrier to T-cells. Endothelial cells may attenuate T-cell activity, target them, and functionally and physically prevent their entry into the tumor in the first place through the deregulation of adhesion molecules. Several therapeutic approaches have been developed to penetrate the tumor endothelial barrier and have further been shown to act synergistically with active adoptive immunotherapies. Taking into account the heterogeneity of solid tumors, well-designed trials are warranted in order to clarify optimal combinations of antiangiogenic drugs and immunotherapeutic agents for each cancer type. For that purpose, monitoring of the changes in tumor vascularity and the patient's pre- and post-treatment immunity, detection of immune biomarkers to measure antiangiogenic responses is essential. Furthermore, the optimal doses and the schedule of treatment modalities should be determined to achieve clinical effectiveness and to avoid serious side effects.

Next-generation sequencing, which encompasses whole genome, whole transcriptome sequencing, whole exome, and targeted sequencing, has substantially expanded our knowledge in cancer biology by identifying mutations with high sensitivity and epitope prediction algorithms, which can predict the binding of mutant peptides. Through the next-generation sequencing, we have discovered that neoantigen-specific T-cell responses may interfere in the efficacy of checkpoint blockade and of the adoptive cell transfer. In the near future, it may be possible to design personalized vaccines encoding predicted neoantigens, stratifying patients for based on the number of predicted antigens in tumors for checkpoint inhibition. It will also be feasible to determine the effective duration and dose of checkpoint blockade by detecting neoantigen T-cell responses. However, although it is intriguing, exome or whole genome sequencing has yet to be incorporated into routine clinical care, and mutational burden does not yet have a role in selecting patients for therapy, because major challenges lie ahead. First, the need exists to identify and track neoantigen reactive T-cells. Second, it is crucial to find out which neoantigens are processed to provide binding peptides presented by cancer cells. Third, the development of therapies that are able to mobilize neoantigen reactive T-cells need to be developed.

The long-term toxicity and the impact of immunotherapy on quality of life should be evaluated in further pharmacogenomics and biological studies in an attempt to get an insight into the patient's immunologic profile and a potential predisposition to the occurrence of adverse events. Also, the safety of immunotherapy in patients with an underlying autoimmune disease is virtually unknown since these patients have been excluded from clinical trials. Aclose collaboration between specialists is warranted to explore the pathophysiology of autoimmune diseases and accordingly to adjust treatment with immunotherapies.

Decades of research has led to an understanding of the molecular pathways involved in malignancies, recognition of the complexity of biological networks, and the clarification of the role of immunotherapy in cancer. The new era of genetic and molecular analyses, including the assessment of the prognostic landscape of genes and infiltrating immune cells as well as the expression of molecularly determined neoantigens by tumors, leading to their increased immunogeneicity, has generated a new understanding of the prognostic importance of the composition of the tumor and its microenvironment. The gap between bench and bed is getting smaller. Optimal combinations and sequencing of treatment modalities needs to be clarified and in the future we will know if it leads to cancer cure and to the conversion of nonimmunogenic tumors into immunogenic tumors responsive to immune-based therapies. 


\section{References}

[1] Martin SD, Coukos G, Holt RA, Nelson BH: Targeting the undruggable: immunotherapy meets personalized oncology in the genomic era. Ann Oncol. 2015; 12: 2357-2374

[2] Apetoh L, Ladiore S, Coukos G, Ghiringhelli F. Combining immunotherapy and anticancer agents: the right path to achieve cancer cure? Ann Oncol. 2015; 9: 1813-1823

[3] Lanitis E, Irving M, Coukos G. Targeting the tumor vasculature to enhance $T$ cell activity. Curr Opin Immunol. 2015; 33: 53-63
[4] Chiang CL, Bajint K, Coukos G, Kandalaft LE. Potential approaches for more successful dendritic cell-based immunotherapy. Expert Opin Ther. 2015; 15: $569-582$

[5] Michot JM, Bigenwald C, Champiat S, Collins M, Carbonnel F, Postel-Vinay S, et al. Immune-related adverse events with immune checkpoint blockade: a comprehensive review. Eur J Cancer. 2016; 54: 139-148 